\title{
Interplay of charge and orbital ordering in manganese perovskites
}

\author{
K. H. Ahn and A. J. Millis \\ Department of Physics and Astronomy, The Johns Hopkins University \\ Baltimore, Maryland 21218
}

\begin{abstract}
A model of localized classical electrons coupled to lattice degrees of freedom and, via the Coulomb interaction, to each other, has been studied to gain insight into the charge and orbital ordering observed in lightly doped manganese perovskites. Expressions are obtained for the minimum energy and ionic displacements caused by given hole and electron orbital configurations. The expressions are analyzed for several hole configurations, including that experimentally observed by Yamada et al. in $\mathrm{La}_{7 / 8} \mathrm{Sr}_{1 / 8} \mathrm{MnO}_{3}$. We find that, although the preferred charge and orbital ordering depend sensitively on parameters, there are ranges of the parameters in which the experimentally observed hole configuration has the lowest energy. For these parameter values we also find that the energy differences between different hole configurations are on the order of the observed charge ordering transition temperature. The effects of additional strains are also studied. Some results for $\mathrm{La}_{1 / 2} \mathrm{Ca}_{1 / 2} \mathrm{MnO}_{3}$ are presented, although our model may not adequately describe this material because the high temperature phase is metallic.
\end{abstract}

71.38.+i, 71.45.Lr, 71.20.Be, 72.15.Gd

Typeset using REVTEX 
Over the last few years much attention has been focused on manganese perovskite-based oxides, most notably the pseudocubic materials $R e_{1-x} A k_{x} \mathrm{MnO}_{3}$. (Here $R e$ is a rare earth element such as La, and $A k$ is a divalent alkali metal element such as Ca or Sr.) The initial motivation came from the observation that for some range of $x$, and temperature, $T$, resistance can be reduced by a factor of up to $10^{7}$ in the presence of a magnetic field. 1 Two other interesting physical phenomena occurring in this class of materials are charge ordering and orbital ordering. In this paper, we study the connection between the two.

The important electrons in $R e_{1-x} A k_{x} \mathrm{MnO}_{3}$ are the $\mathrm{Mn} e_{g}$ electrons; their concentration is $1-x$. For many choices of $R e, A k$, and $x$, especially at commensurate $x$ values, the $e_{g}$ charge distribution is not uniform and it indeed appears that a fraction $x$ of Mn ions have no $e_{g}$ electron while $1-x$ have a localized $e_{g}$ electron. A periodic pattern of filled and empty sites is said to exhibit charge ordering. There are two $e_{g}$ orbitals per Mn ion. A localized Mn $e_{g}$ electron will be in one linear combination of these; a periodic pattern of orbital occupancy is said to exhibit orbital ordering. Recently, Murakami et al.3 observed the charge ordering transition accompanying simultaneous orbital ordering in $\mathrm{La}_{1 / 2} \mathrm{Sr}_{3 / 2} \mathrm{MnO}_{4}$ at $217 \mathrm{~K}$ (well above the magnetic phase transition temperature $110 \mathrm{~K}$ ). It indicates that the interplay of the charge and orbital ordering to minimize the lattice energy could be the origin of the charge ordering. In this paper we present an expression for the coupling between charge and orbital ordering, with different charge ordering patterns favoring different orbital orderings. We also argue that the orbital ordering energy differences determine the observed charge ordering in lightly doped manganites. Localized charges induce local lattice distortions, which must be accommodated into the global crystal structure; the energy cost of this accommodation is different for different charge ordering patterns.

To model the charge and orbital ordering, we assume that the electrons are localized classical objects, so that each Mn site is occupied by zero or one $e_{g}$ electron, and each $e_{g}$ electron is in a definite orbital state. This assumption seems reasonable in the lightly doped materials such as $\mathrm{La}_{7 / 8} \mathrm{Sr}_{1 / 8} \mathrm{MnO}_{3}$, which are strongly insulating at all temperatures, 1 but may not be reasonable for the $\mathrm{La}_{1 / 2} \mathrm{Ca}_{1 / 2} \mathrm{MnO}_{3}$ composition, 2 where the charge ordered state emerges at a low temperature from a metallic state. We proceed by calculating the energies of different charge ordering patterns, emphasizing the $1 / 8$ doping case. It is practically impossible to consider all possible charge ordering configurations. Therefore, we consider the three configurations shown in Fig. 1, which are the only ones consistent with the following basic features of the hole-lattice implied by the experimental results by Yamada et al. 1 : invariance under translation by two lattice constants in the $x$ or $y$ direction, four in the $z$ direction, and an alternating pattern of occupied and empty planes along $z$ direction. The configuration in Fig. 1(b) is the one proposed by Yamada et al. $\mathbf{\theta}_{\text {to }}$ explain their experimental results for $\mathrm{La}_{7 / 8} \mathrm{Sr}_{1 / 8} \mathrm{MnO}_{3}$. For localized electrons there are three energy terms : the coupling to the lattice, which will be discussed at length below, the Coulomb interaction, and the magnetic interaction.

First, we argue that the Coulomb energy cannot explain the observed ordering pattern or transition temperature. We take as reference the state with one $e_{g}$ electron per Mn and denote by $\delta q_{i}$ the charge of a hole on a Mn site. From the classical Coulomb energy

$$
U_{\text {Coulomb }}=\frac{1}{2 \epsilon_{0}} \sum_{i \neq j} \frac{\delta q_{i} \delta q_{j}}{r_{i j}},
$$


one finds that the difference in energy between the configurations in Fig. 11 is

$$
\Delta \mathcal{U}_{\text {Coulomb, per hole }}=\frac{1}{2 \epsilon_{0}} \sum_{i \neq o} \frac{\Delta\left(\delta q_{i}\right)}{r_{i o}}
$$

where $o$ is a site containing a hole and $\Delta(\delta q)$ is the difference in charge between the two configurations. We estimated the above infinite sum by repeated numerical calculations for larger and larger volumes of the unit cells around the origin. We find that Fig. 11(c) has the lowest energy; $12 \mathrm{meV} / \epsilon_{o}$ lower than Fig. 11(b), and $27 \mathrm{meV} / \epsilon_{o}$ lower than Fig. 11(a).

To estimate the magnitude of the Coulomb energy differences, we need an estimate for the dielectric constant $\epsilon_{0}$, which we obtain from the measured reflectivity for $\mathrm{La}_{0.9} \mathrm{Sr}_{0.1} \mathrm{MnO}_{3}$, and the Lyddane-Sachs-Teller relation $\omega_{L}^{2}=\omega_{T}^{2} \epsilon_{0} / \epsilon_{\infty}$. At frequencies greater than the greatest phonon frequency the reflectivity is close to 0.1 , implying $\epsilon_{\infty} \approx 3.4$; the reflectivity is near unity between $\omega_{T}=0.020 \mathrm{eV}$, and $\omega_{L}=0.024 \mathrm{eV}$, implying $\epsilon_{0} \approx 5.0$. Because both $\mathrm{La}_{7 / 8} \mathrm{Sr}_{1 / 8} \mathrm{MnO}_{3}$ and $\mathrm{La}_{0.9} \mathrm{Sr}_{0.1} \mathrm{MnO}_{3}$ are insulating and have similar compositions, their static dielectric constants are expected to be similar. Using $\epsilon_{0} \approx 5.0$, the energy difference between different configurations of holes is only around $2.4 \mathrm{meV}$, or $30 \mathrm{~K}$ per hole, which is small compared to the observed charge ordering temperature of $150 \mathrm{~K}-200 \mathrm{~K}$ of these materials. The inconsistency with the experimentally observed hole configuration and the smallness of the energy difference scale indicate that the electrostatic energy is not the main origin of charge ordering for this material.

Even though the magnetic and charge ordering transitions show a correlation in $\mathrm{La}_{7 / 8} \mathrm{Sr}_{1 / 8} \mathrm{MnO}_{3}$, , we do not think that the magnetic contribution to charge and orbital ordering is as important as the lattice contribution for three reasons. First, in undoped $\mathrm{LaMnO}_{3}$, the orbital ordering and the structural phase transition occur at around $800 \mathrm{~K}$ and the magnetic ordering at around $140 \mathrm{~K}$, 8 suggesting that the magnetic effects are relatively weak. Second, in $\mathrm{La}_{7 / 8} \mathrm{Sr}_{1 / 8} \mathrm{MnO}_{3}$ the $\mathrm{Mn}$ spins are ferromagnetically ordered with moment close to the full $\mathrm{Mn}$ moment at temperatures greater than the charge ordering temperature,, and ferromagnetic order does not favor one charge configuration over another. Third, although in $\mathrm{La}_{7 / 8} \mathrm{Sr}_{1 / 8} \mathrm{MnO}_{3}$ antiferromagnetic order appears at the charge ordering transition, the antiferromagnetic moment is very small (less than 0.1 of the full $\mathrm{Mn}$ moment), so the energy associated with this ordering must be much less than $140 \mathrm{~K} /$ site associated with magnetic ordering in $\mathrm{LaMnO}_{3}$. Therefore, we think that the canted antiferromagnetism occuring upon charge ordering in $\mathrm{La}_{7 / 8} \mathrm{Sr}_{1 / 8} \mathrm{MnO}_{3}$ (Ref. 7) is not the cause but the effect of the charge and orbital ordering. We now turn our attention to the lattice energy.

A classical model for the lattice distortions of the insulating perovskite manganites has been derived in Ref. 10, and shown to be consistent with experimental results on $\mathrm{LaMnO}_{3}$. This model is adopted here with an additional term, an energy cost for shear strain. We now briefly outline the model, which is explained in more detail in Ref. 10 and the Appendix. The ionic displacements included are the vector displacement $\vec{\delta}_{i}$ of the Mn ion on site $i$, and the $\hat{a}$ directional scalar displacement $u_{i}^{a}(a=x, y$, and $z)$ of the $\mathrm{O}$ ion which sits between the Mn ion on site $i$ and the Mn ion on site $i+\hat{a}$. For convenience, $\vec{\delta}_{i}$ and $u_{i}^{a}$ are defined to be dimensionless in the following way: the lattice constant of the ideal cubic perovskite is $b$, the Mn ion position in the ideal cubic perovskite is $\vec{R}_{i}$, the actual Mn ion 
position is $\vec{R}_{i}+b \vec{\delta}_{i}$, and the actual O ion position is $\vec{R}_{i}+\left(b / 2+b u_{i}^{a}\right) \hat{a}$. The lattice energy is taken to be harmonic and depends only on the nearest neighbor Mn-O distance and the first and second nearest neighbor Mn-Mn distances. The spring constants corresponding to these displacements are $K_{1}, K_{2}$, and $K_{3}$ as shown in Fig. 2. Because $K_{1}$ and $K_{2}$ involve bond stretching, while $K_{3}$ involves bond bending, $K_{1} \geq K_{2} \gg K_{3}$ is expected. Thus, $E_{\text {lattice }}=E_{\mathrm{Mn}-\mathrm{O}}+E_{\mathrm{Mn}-\mathrm{Mn} \text {,first }}+E_{\mathrm{Mn}-\mathrm{Mn} \text {,second }}$, where

$$
\begin{aligned}
E_{\mathrm{Mn}-\mathrm{O}}= & \frac{1}{2} K_{1} \sum_{i, a}\left(\delta_{i}^{a}-u_{i}^{a}\right)^{2}+\left(\delta_{i}^{a}-u_{i-a}^{a}\right)^{2} \\
E_{\mathrm{Mn}-\mathrm{Mn}, \mathrm{first}}= & \frac{1}{2} K_{2} \sum_{i, a}\left(\delta_{i}^{a}-\delta_{i-a}^{a}\right)^{2}, \\
E_{\mathrm{Mn}-\mathrm{Mn}, \text { second }}= & \frac{1}{2} K_{3} \sum_{i,(a, b)}\left[\left(\frac{\delta_{i+a+b}^{a}+\delta_{i+a+b}^{b}}{\sqrt{2}}\right)-\left(\frac{\delta_{i}^{a}+\delta_{i}^{b}}{\sqrt{2}}\right)\right]^{2} \\
& +\left[\left(\frac{\delta_{i+a-b}^{a}-\delta_{i+a-b}^{b}}{\sqrt{2}}\right)-\left(\frac{\delta_{i}^{a}-\delta_{i}^{b}}{\sqrt{2}}\right)\right]^{2} .
\end{aligned}
$$

In the above equations $a$ denotes $x, y$, and $z$, and $(a, b)$ represents $(x, y),(y, z)$, and $(z, x)$. $E_{\mathrm{Mn}-\mathrm{Mn} \text {,second }}$ was not considered in Ref. 10. The shear modulus produced by this term is important, because without it, a $\mathrm{Mn}$ ion on site $i+\hat{x}$ can have arbitrary large $y$ directional displacement relative to the $\mathrm{Mn}$ ion on site $i$ at no cost of energy. For this reason, the model with $K_{3}=0$ has singularities, whose proper treatment requires $K_{3} \neq 0$ in our model. However, still we expect $K_{3}$ will be much smaller than $K_{1}$ or $K_{2}$. Therefore, in order to simplify the calculation, the $K_{3} / K_{1} \rightarrow 0$ limit has been taken after the expression of minimized energy and equilibrium ionic displacements have been obtained.

Second, we consider the electronic degree of freedom. We parameterize the electron density by the variable $h_{i}$. If an electron is present on site $i, h_{i}=0$; if no electron is present, $h_{i}=1$. If there is an electron on site $i$, the electron orbital state, which is a linear combination of the two $e_{g}$ orbitals, is parameterized by an angle $\theta_{i}$ as follows.

$$
\left|\psi_{i}\left(\theta_{i}\right)>=\cos \theta_{i}\right| d_{3 z^{2}-r^{2}}>+\sin \theta_{i} \mid d_{x^{2}-y^{2}}>
$$

with $0 \leq \theta_{i}<\pi$. The electron orbital state couples to the distortion of the surrounding oxygen octahedra through the Jahn-Teller distortion. The coupling is given by

$$
\begin{aligned}
E_{\mathrm{JT}} & =-\lambda \sum_{i}\left(1-h_{i}\right)\left[\cos 2 \theta_{i}\left\{v_{i}^{z}-\frac{1}{2}\left(v_{i}^{x}+v_{i}^{y}\right)\right\}+\sin 2 \theta_{i} \frac{\sqrt{3}}{2}\left(v_{i}^{x}-v_{i}^{y}\right)\right] \\
& =-\lambda \sum_{i, a}\left(1-h_{i}\right) v_{i}^{a} \cos 2\left(\theta_{i}+\psi_{a}\right)
\end{aligned}
$$

where

$$
\begin{aligned}
v_{i}^{a} & =u_{i}^{a}-u_{i-a}^{a}, \\
\psi_{x}=-\pi / 3, \psi_{y} & =\pi / 3, \psi_{z}=0 .
\end{aligned}
$$

If a hole is present on site $i$, it attracts the surrounding oxygens equally, giving rise to a breathing distortion energy given by 


$$
E_{\mathrm{hole}}=\beta \lambda \sum_{i} h_{i}\left(v_{i}^{x}+v_{i}^{y}+v_{i}^{z}\right)
$$

The parameter $\beta$ represents the strength of the breathing distortion relative to the JahnTeller distortion. Finally, following Kanamori, ${ }^{\mathbb{Q}}$ we include a phenomenological cubic anharmonicity term given by

$$
E_{\text {anharm }}=-A \sum_{i}\left(1-h_{i}\right) \cos 6 \theta_{i} .
$$

The sign has been chosen so that the electron orbital states of $\left|3 x^{2}-r^{2}\right\rangle,\left|3 y^{2}-r^{2}\right\rangle$, or $\mid 3 z^{2}-r^{2}>$, with $\hat{x}, \hat{y}$, and $\hat{z}$ pointing toward nearest oxygen ions are favored when $A$ is positive. The total energy, which is the sum of all the above energy terms, is given by

$$
E_{\mathrm{tot}}=E_{\mathrm{Mn}-\mathrm{O}}+E_{\mathrm{Mn}-\mathrm{Mn}, \mathrm{first}}+E_{\mathrm{Mn}-\mathrm{Mn}, \text { second }}+E_{\mathrm{JT}}+E_{\text {hole }}+E_{\text {anharm }} .
$$

We minimized $E_{\text {tot }}$ about $\delta_{i}^{a}$ 's and $u_{i}^{a}$ 's for fixed hole and orbital configurations. These are conveniently expressed in terms of the variables $\delta_{\vec{k}}^{a}, u_{\vec{k}}^{a}, h_{\vec{k}}$, and $c_{\vec{k}}^{a}$ defined in the following way.

$$
\begin{aligned}
\delta_{i}^{a} & =\sum_{\vec{k}} e^{-i \vec{k} \cdot \vec{R}_{i}} \delta_{\vec{k}}^{a}, \\
u_{i}^{a} & =\sum_{\vec{k}} e^{-i \vec{k} \cdot \vec{R}_{i}} u_{\vec{k}}^{a}, \\
h_{i} & =\sum_{\vec{k}} e^{-i \vec{k} \cdot \vec{R}_{i}} h_{\vec{k}}, \\
\left(1-h_{i}\right) \cos 2\left(\theta_{i}+\psi_{a}\right) & =\sum_{\vec{k}} e^{-i \vec{k} \cdot \vec{R}_{i}} c_{\vec{k}}^{a} .
\end{aligned}
$$

The details are shown in the Appendix. The minimized energy per Mn ion may be written as

$$
\frac{E_{\text {tot }}}{N}=\mathcal{E}_{\vec{k}=0}+\sum_{\vec{k} \neq 0, a} \mathcal{E}_{\vec{k}}^{a}+\frac{E_{\text {anharm }}}{N}
$$

where

$$
\begin{aligned}
\mathcal{E}_{\vec{k}}^{a} & = \begin{cases}-\frac{\lambda^{2}}{\left(K_{1}+2 K_{2}\right) K_{1}}\left[K_{1}+K_{2}\left(1-\cos k_{a}\right)\right]\left(\beta h_{\vec{k}}-c_{\vec{k}}^{a}\right)\left(\beta h_{-\vec{k}}-c_{-\vec{k}}^{a}\right), & \text { if } k_{a} \neq 0 \\
0, & \text { if } k_{a}=0\end{cases} \\
\mathcal{E}_{\vec{k}=0} & =-\frac{\lambda^{2}}{K_{1}+2 K_{2}}\left[3\left(\beta h_{0}\right)^{2}+\sum_{a}\left(c_{0}^{a}\right)^{2}\right] .
\end{aligned}
$$

The long wave length strain $e^{a b}$, and the $\vec{k}(\neq 0)$ components of the ionic displacements are given as

$$
\begin{aligned}
e^{a b} & =-\frac{2 \lambda}{K_{1}+2 K_{2}}\left(\beta h_{0}-c_{0}^{a}\right) \delta_{a b}, \\
u_{\vec{k} \neq 0}^{a} & = \begin{cases}-\frac{\lambda\left[K_{1}+K_{2}\left(1-\cos k_{a}\right)\right]}{\left(K_{1}+2 K_{2}\right) K_{1}} \frac{1-e^{-i k_{a}}}{1-\cos k_{a}}\left(\beta h_{\vec{k}}-c_{\vec{k}}^{a}\right), & \text { if } k_{a} \neq 0 \\
0, & \text { if } k_{a}=0\end{cases} \\
\delta_{\vec{k} \neq 0}^{a} & = \begin{cases}-i \frac{\lambda \sin k_{a}}{\left(K_{1}+2 K_{2}\right)\left(1-\cos k_{a}\right)}\left(\beta h_{\vec{k}}-c_{\vec{k}}^{a}\right), & \text { if } k_{a} \neq 0 \\
0, & \text { if } k_{a}=0 .\end{cases}
\end{aligned}
$$


Because $h_{i}$ 's and $\left(1-h_{i}\right) \cos 2\left(\theta_{i}+\psi_{a}\right)$ 's are bounded by \pm 1 , we cannot treat $h_{\vec{k}}$ 's and $c_{\vec{k}}^{a}$ 's as independent variables to minimize $E_{\text {tot }}$. Therefore, we minimize $E_{\text {tot }}$ over the orbital variable $\theta_{i}$ 's at fixed hole configurations; the ground state is then the hole configuration of the lowest energy.

For $\mathrm{La}_{7}{ }_{8} \mathrm{Sr}_{1 / 8} \mathrm{MnO}_{3}$, we consider the three hole configurations shown in Fig. 1, each of which is a Bravais lattice, with a unit cell containing one Mn site with a hole and seven Mn sites without holes. The orbital configuration may be different in different unit cells of the lattice defined by the holes. We consider the case where the orbital configuration is the same in each unit cell. In addition to that, we also consider all possible two sublattice symmetry breakings. Therefore, we have seven (if no symmetry breaking) or fourteen (if two-sublattice symmetry breaking) orbital variable $\theta_{i}$ 's. $E_{\text {tot }} / N$ in Eq. (17) for each configuration is expressed in terms of those variables through Eqs. (11), (15), (16), (18), and (19), and is minimized about $\theta_{i}$ 's. For this minimization, we use the FindMinimum routine in Mathematica in the following way: for each set of parameters, and for each configuration, we check the local minimal values by using $50-200$ random starting values of $\theta_{i}$ 's.

According to Ref. $10, \lambda / K_{1}$ ranges over $0.04-0.05$, and $K_{2} / K_{1}$ is between 0 and 1 . $A / K_{1}$ ranges around 0.0002 , and $K_{1} \approx 200 \mathrm{eV} 10$ Recently, a local breathing distortion of $0.12 \AA$ has been directly observed in $\mathrm{La}_{0.75} \mathrm{Ca}_{0.25} \mathrm{MnO}_{3}$. 11 The Jahn-Teller distortion is estimated around $0.15 \AA$ from the $\mathrm{Mn}-\mathrm{O}$ distances of $\mathrm{LaMnO}_{3} .2$ This implies that the breathing distortion and the Jahn-Teller distortion in these materials have similar order of magnitude, i.e., $\beta=O(1)$. We varied $\beta$ in the range of $0-10$, and $A / K_{1}$ in the range of $0-0.00035$, with $\lambda / K_{1}=0.045, K_{2} / K_{1}=0.5$, and $K_{1}=200 \mathrm{eV}$. For each set of those parameters, the minimum energy per hole for each fixed hole configuration in Fig. [1 has been found. By comparing them, we find the most favored hole configuration for each $\beta$ and $A / K_{1}$, which is shown in Fig. 通 as a plot in $\beta-A / K_{1}$ plane.

At large $\beta(\gtrsim 7)$, the configuration shown in Fig. $\mathbb{1}(\mathrm{c})$ is the most favored, and that shown in Fig. 1(a) is the least favored. This can be related to the fact that, in $y-z$ and $z-x$ directional planes, Fig. [1(c) has the most even distribution of holes, and Fig. [1(a) has the least even distribution. For large $\beta$, the contraction of oxygen octahedra toward holes is strong, and an uneven distribution of holes generates larger strains and elevates minimum energies. Particularly, the square hole net squeezes the electron orbital at the center of the square along the direction perpendicular to the square plane. In the cubic hole configuration of Fig. [1(a), the six squeezed electron orbitals point toward the cubic center, putting the electron orbital at the center at high energy, which is consistent with our result that Fig. [1(a) has far higher minimum energies than Figs. [1(b) and [1(c) in large $\beta$ limit.

As $\beta$ is decreased into the range of $2-5$, the favored hole configuration becomes that of Fig. 1(b), which is the experimentally observed hole configuration. We expect that the difference of the energy per hole between the ground state hole configuration and the next lowest energy hole configuration corresponds approximately to the charge ordering temperature. The calculation results indicate that, when $\beta$ is in the range of $2.0-2.5$ or around 5.0 and $A / K_{1}=0.0002$, the charge ordering temperature is around $100-200 \mathrm{~K}$, which is consistent with experimental results. As $\beta$ is decreased further, the most favored hole configuration changes further and the temperature difference scale decreases.

Figure 3 also shows the tendency that the configuration of Fig. 1(c) becomes more favored as $A / K_{1}$ increases. We think this occurs because the anharmonicity energy distorts the 
oxygen octahedra tetragonally, which can be more easily accommodated by the tetragonal hole configuration of Fig. 1)(c).

In Table [I, we have shown an example of the orbital states, ionic displacements, and uniform strains corresponding to the minimum energy configuration for Fig. 1(b) when $A / K_{1}=0.0002, \lambda / K_{1}=0.045, K_{2} / K_{1}=0.5$ and $\beta=2.5 . x, y$, and $z$ directions are shown in Fig. 1. The nearest Mn-Mn distance is unit. $\left(n_{i}^{x}, n_{i}^{y}, n_{i}^{z}\right)$ is defined in such a way that $\left(n_{i}^{x}, n_{i}^{y}, n_{i}^{z}\right)+N_{1}(2,0,0)+2 N_{2}(0,2,0)+2 N_{3}(1,0,2)$ 's and $\left(n_{i}^{x}, n_{i}^{y}, n_{i}^{z}\right)+N_{1}(2,0,0)+\left(2 N_{2}+\right.$ 1) $(0,2,0)+\left(2 N_{3}+1\right)(1,0,2)$ 's, where $N_{1}, N_{2}$, and $N_{3}$ are integers, represent the coordinates of the sites indexed by $i . \vec{k}=0$ parts of the ionic displacements have been subtracted to find the non-uniform parts of the displacements.

The energy expressions in Eqs. (18) and (19) are adequate for bulk materials. When the material is grown on a substrate as a thin film, generally there is a strain generated by lattice mismatch between the film and the substrate materials. To see the effect of this strain, we add a term proportional to $c_{0}^{a^{\prime}}\left(a^{\prime}=x, y\right.$, or $\left.z\right)$ to the energy, which corresponds to an $a^{\prime}$ directional strain. Using a parameter $g$, we replace $\mathcal{E}_{\vec{k}=0}$ in Eq. (19) by the following expression:

$$
\mathcal{E}_{\vec{k}=0}^{\prime}=-\frac{\lambda^{2}}{K_{1}+2 K_{2}}\left[3\left(\beta h_{0}\right)^{2}+g c_{0}^{a^{\prime}}+\sum_{a}\left(c_{0}^{a}\right)^{2}\right]
$$

We repeated similar calculations to find the favored hole configurations for different values of the applied strain, parameterized by $g$. The applied strain breaks cubic symmetry. Some of the hole configurations also break cubic symmetry. For these cases the energy depends on the relative orientation of the strain and hole symmetry breakings. We consider all possible orientations and find the lowest energy state. We have varied $g$ between -0.4 and 0.4 , and $\beta$ between 0 and 7 , with $A / K_{1}=0.0002, \lambda / K_{1}=0.045$, and $K_{2} / K_{1}=0.5$. The results are shown as a phase diagram in $\beta-g$ plane in Fig. A. It shows that Fig. 1)(c) configuration is favored more as $|g|$ increases. This feature can be understood in the following way. For small $g$ 's, the leading correction to the minimum energy for each hole configuration is $-\lambda^{2} g \tilde{c}_{0}^{a} /\left(K_{1}+2 K_{2}\right)$, where $\tilde{c_{0}^{a}}$ represents $\left.c_{0}^{a}\right|_{g=0}$. Therefore the configuration which has a larger $\left|\tilde{c}_{0}^{a}\right|$ will show greater change in energy for a given $g$. Since the hole configuration in Fig. 11(c) has tetragonal symmetry, which is compatible with the Jahn-Teller distortion, it has the largest $\left|\tilde{c_{0}^{a}}\right|$. Therefore, as $|g|$ increases, Fig. 11(c) is more favored than Figs. 1(a) and 1(b). Because the energy changes linearly with $g$, the phase boundaries are straight lines for small $g$, and have cusps at $g=0$, as shown in Fig. 4 . Typical variations of $e^{a a}$ corresponding to changing $|g|$ from 0 to 0.4 are about $2 \%$. The results indicate that the strain generated by substrates can change ordered hole configuration and ordering temperature.

Our results indicate that the interaction of the electronic state and the lattice can be the origin of the charge ordering in this material, even though the details of the results are dependent on specific choice of $K_{1}, K_{2}, \lambda$, and $A$.

A similar calculation has been done for $R e_{1 / 2} A k_{1 / 2} \mathrm{MnO}_{3}$, hole concentration $1 / 2$. We choose the three hole configurations in Fig. 5 to compare the minimum energies. Each configuration has an alternating hole distribution in different set of directions : $x, y$, and $z$ directions for Fig. 5(a), $x$ and $y$ directions for Fig. 5(b), and $y$ direction for Fig. 5(c). Figure 5 (b) is the experimentally observed hole configuration.2 As we have done for $x=1 / 8$, we 
consider both the case where hole and orbital state have the same unit cell, and the case where the orbital state is composed of the two hole sublattices. Calculations for $A / K_{1}=$ $0.0002, \lambda / K_{1}=0.045$, and $K_{2} / K_{1}=0.5$ show that when $\beta$ is large, configuration in Fig. 5(a) is the most favored and Fig. 5(c) is the least favored. As $\beta$ is decreased, the favored configuration is changed between $\beta=0.5$ and 0.7. After that Fig. 5(c) is the most favored and Fig. 5(a) is the least favored. When $\beta$ is large, the holes prefer to distribute evenly because of the same reason as in $x=1 / 8$ case. In contrast, when $\beta$ is small, electron sites prefer to have more neighboring electron sites to gain orbital energy. Our results are not consistent with the experimental results for $\mathrm{La}_{1 / 2} \mathrm{Ca}_{1 / 2} \mathrm{MnO}_{3}$, which indicate that configuration Fig.

5(b) is the ground state. This inconsistency may arise because our model involves only localized electrons, while for $x=1 / 2$ the charge ordering state arises from a metallic phase. Modifications of our model to include hole hopping are desirable.

In summary, we have shown that the lattice effect could play an important role in the charge ordering transition observed in perovskite manganites.

\section{ACKNOWLEDGMENTS}

This work was supported in part by NSF-DMR-9705482. We also acknowledge partial support from NSF-DMR-96322526 (The Johns Hopkins M.R.S.E.C. for Nanostructured materials).

\section{APPENDIX:}

To find the minimum energy we transform $E_{\text {tot }}$ in Eq. (12) into $\vec{k}$ space, using Eqs. (13) - (16). This leads to the following energy expressions in $k$ space:

$$
\begin{aligned}
E_{\mathrm{tot}} /\left(N K_{1}\right)= & \sum_{\vec{k}} \delta_{\vec{k}}^{\dagger} M_{\vec{k}} \delta_{\vec{k}}+\delta_{\vec{k}}^{\dagger} L_{\vec{k}}^{\dagger} u_{\vec{k}}+u_{\vec{k}}^{\dagger} L_{\vec{k}} \delta_{\vec{k}}+u_{\vec{k}}^{\dagger} u_{\vec{k}}+u_{\vec{k}}^{\dagger} P_{\vec{k}} e_{\vec{k}}+e_{\vec{k}}^{\dagger} P_{\vec{k}}^{\dagger} u_{\vec{k}} \\
& -\frac{A}{N K_{1}} \sum_{i}\left(1-h_{i}\right) \cos 6 \theta_{i},
\end{aligned}
$$

where

$$
\begin{aligned}
\delta_{\vec{k}}^{\dagger} & =\left(\delta_{\vec{k}}^{x}, \delta_{\vec{k}}^{y}, \delta_{\vec{k}}^{z}\right), \\
u_{\vec{k}}^{\dagger} & =\left(u_{\vec{k}}^{x}, u_{\vec{k}}^{y}, u_{\vec{k}}^{z}\right), \\
e_{\vec{k}}^{\dagger} & =\left(\beta h_{\vec{k}}-c_{\vec{k}}^{x}, \beta h_{\vec{k}}-c_{\vec{k}}^{y}, \beta h_{\vec{k}}-c_{\vec{k}}^{z}\right),
\end{aligned}
$$




$$
M_{\vec{k}}=\left(\begin{array}{ccc}
1+\frac{K_{2}}{K_{1}}\left(1-\cos k_{x}\right) & \\
+\frac{K_{3}}{K_{1}}\left(1-\cos k_{x} \cos k_{y}\right) & \frac{K_{3}}{K_{1}} \sin k_{x} \sin k_{y} & \frac{K_{3}}{K_{1}} \sin k_{x} \sin k_{z} \\
+\frac{K_{3}}{K_{1}}\left(1-\cos k_{x} \cos k_{z}\right) & 1+\frac{K_{2}}{K_{1}}\left(1-\cos k_{y}\right) \\
\frac{K_{3}}{K_{1}} \sin k_{y} \sin k_{x} & +\frac{K_{3}}{K_{1}}\left(1-\cos k_{y} \cos k_{z}\right) \\
+\frac{K_{3}}{K_{1}}\left(1-\cos k_{y} \cos k_{x}\right) & \frac{K_{3}}{K_{1}} \sin k_{y} \sin k_{z} \\
\frac{K_{3}}{K_{1}} \sin k_{z} \sin k_{x} & \frac{K_{3}}{K_{1}} \sin k_{z} \sin k_{y} & +\frac{K_{3}}{K_{1}}\left(1-\cos k_{z} \cos k_{x}\right) \\
L_{\vec{k}}= & +\frac{K_{3}}{K_{1}}\left(1-\cos k_{z} \cos k_{y}\right)
\end{array}\right),
$$

and $N$ is the total number of Mn sites. We obtain Eqs. (18) - (22) by minimizing the above expression with respect to all $\delta_{\vec{k}}^{a}$ and $u_{\vec{k}}^{a}$. Without the second neighbor elastic energy term, $\delta_{\vec{k}}$ and $u_{\vec{k}}$ minimizing Eq. (A1) become singular when any of $k_{x}, k_{y}$, and $k_{z}$ is zero. With nonzero $K_{3}$ this singularity has been uniquely solved for $\vec{k} \neq 0$, while at $\vec{k}=0$, it is not.

To find the energy term with $\vec{k}=0$, we take the $\vec{k} \rightarrow 0$ limit. That corresponds to the uniform strain energy, i.e., the energy related to the change of the lattice parameters from the original cubic structure. Here the problem of the choice of the limiting process arises, because the calculation shows that the different directions of the limiting process of $\vec{k} \rightarrow 0$ have given different energies and different uniform strains. Because the lower energy state is favored after all, the appropriate limiting process will be the one which gives the minimum uniform strain energy, and it determines the uniform strain also. When $K_{3} / K_{1} \ll 1$, this appropriate limiting process has been found to satisfy the condition of $k_{x}, k_{y}$, and $k_{z} \neq 0$. As far as $k_{x}, k_{y}$, and $k_{z}$ are nonzero, the limits are different only in the order of $K_{3} / K_{1}$. Therefore, in the $K_{3} / K_{1} \rightarrow 0$ limit, any $\vec{k} \rightarrow 0$ limit process satisfying the above condition gives the correct expression of the minimum energy term with $\vec{k}=0$. It also gives a unique uniform strain. 


\section{REFERENCES}

${ }^{1}$ R. von Helmolt, J. Wecker, B. Holzapfel, L. Schultz, and K. Samwer, Phys. Rev. Lett. 71, 2331 (1993); S. Jin, T. H. Tiefel, M. McCormack, R. A. Fastnacht, R. Ramesh, and L. H. Chen, Science 264, 413 (1994); K. Liu, X. W. Wu, K. H. Ahn, T. Sulchek, C. L. Chien, and J. Q. Xiao, Phys. Rev. B 54, 3007 (1996).

${ }^{2}$ C. H. Chen and S-W. Cheong, Phys. Rev. Lett. 76, 4042 (1996).

${ }^{3}$ Y. Murakami, H. Kawada, H. Kawata, M. Tanaka, T. Arima, Y. Moritomo, and Y. Tokura, Phys. Rev. Lett. 80, 1932 (1998).

${ }^{4}$ Y. Yamada, O. Hino, S. Nohdo, and R. Kano, Phys. Rev. Lett. 77, 904 (1996).

${ }^{5}$ Y. Okimoto, T. Katsufuji, T. Ishikawa, T. Arima and Y. Tokura, Phys. Rev. B 55, 4206 (1997).

${ }^{6}$ N. W. Aschcroft, and N. D. Mermin, Solid State Physics (Saunders College Publishing, Fort Worth, TX, 1976).

${ }^{7}$ H. Kawano, R. Kajimoto, M. Kubota, and H. Yoshizawa, Phys. Rev. B 53, 2202 (1996); 53, 14709 (1996).

${ }^{8}$ E. O. Wollan and W. C. Koehler, Phys. Rev. 100, 545 (1955).

9 J. Kanamori, J. Appl. Phys. 31, 14S (1960).

${ }^{10}$ A. J. Millis, Phys. Rev. B 53, 8434 (1996).

11 S. J. L. Billinge, R. G. DiFrancesco, G. H. Kwei, J. J. Neumeier, and J. D. Thompson, Phys. Rev. Lett. 77, 715 (1996)

12 J. B. A. A. Ellemans, B. van Laar, K. R. van der Veen, and B. O. Loopstra, J. Solid State Chem. 3, 238 (1971). 


\section{FIGURES}

FIG. 1. The three hole ordering patterns for $R e_{7 / 8} A k_{1 / 8} \mathrm{MnO}_{3}$ considered in our calculations. Solid circles represent $\mathrm{Mn}^{3+}$, and open circles $\mathrm{Mn}^{4+}$.

FIG. 2. Spring constants: $K_{1}$ between the nearest neighbor Mn-O, $K_{2}$ between the first neighbor Mn-Mn, and $K_{3}$ between the second neighbor Mn-Mn.

FIG. 3. Phase diagram in $A / K_{1}$ versus $\beta$ plane for $R e_{7 / 8} A k_{1 / 7} \mathrm{MnO}_{3} . \quad \lambda / K_{1}=0.045$, $K_{2} / K_{1}=0.5$, and $g=0$.

FIG. 4. Phase diagram in $g$ versus $\beta$ plane for $R e_{7 / 8} A k_{1 / 7} \mathrm{MnO}_{3} . \lambda / K_{1}=0.045, K_{2} / K_{1}=0.5$, and $A / K_{1}=0.0002$.

FIG. 5. The three hole ordering patterns for $R e_{1 / 2} A k_{1 / 2} \mathrm{MnO}_{3}$ considered in our calculations. Solid circles represent $\mathrm{Mn}^{3+}$, and open circles $\mathrm{Mn}^{4+}$. 


\section{TABLES}

TABLE I. Coordinates of site $i$, orbital states, ionic displacements, and uniform strains for the minimum energy configuration of Fig. 1 1 (b), when $A / K_{1}=0.0002, \lambda / K_{1}=0.045, K_{2} / K_{1}=0.5$ and $\beta=2.5$.

\begin{tabular}{c|c|c|c|c|c|c|c|c}
\hline \hline $\mathrm{i}$ & $\left(n_{i}^{x}, n_{i}^{y}, n_{i}^{z}\right)$ & $\theta_{i}($ radian $)$ & $\delta_{i}^{x}-\delta_{\vec{k}=0}^{x}$ & $\delta_{i}^{y}-\delta_{\vec{k}=0}^{y}$ & $\delta_{i}^{z}-\delta_{\vec{k}=0}^{z}$ & $u_{i}^{x}-u_{\vec{k}=0}^{x}$ & $u_{i}^{y}-u_{\vec{k}=0}^{y}$ & $u_{i}^{z}-u_{\vec{k}=0}^{z}$ \\
\hline 1 & $(0,0,0)$ & hole site & 0 & 0 & 0 & -0.135 & -0.134 & -0.159 \\
2 & $(1,0,0)$ & 1.11 & 0 & 0.007 & 0 & 0.135 & 0.007 & -0.039 \\
3 & $(0,1,0)$ & 1.97 & 0 & 0 & 0 & 0.004 & 0.134 & -0.030 \\
4 & $(1,1,0)$ & 0.03 & 0 & 0 & 0 & -0.004 & -0.007 & 0.047 \\
5 & $(0,0,1)$ & 0.09 & 0 & -0.005 & -0.049 & 0 & -0.023 & 0.011 \\
6 & $(1,0,1)$ & 0.09 & 0 & 0.005 & -0.007 & 0 & -0.009 & 0.019 \\
7 & $(0,1,1)$ & 2.74 & 0 & 0 & 0.002 & -0.043 & 0.023 & 0.037 \\
8 & $(1,1,1)$ & 1.24 & 0 & 0 & 0.013 & 0.043 & 0.009 & -0.008 \\
9 & $(0,2,0)$ & hole site & 0 & 0 & 0 & -0.135 & -0.133 & -0.159 \\
10 & $(1,2,0)$ & 1.11 & 0 & -0.007 & 0 & 0.135 & -0.013 & -0.039 \\
11 & $(0,3,0)$ & 2.28 & 0 & 0 & 0 & -0.036 & 0.133 & 0.008 \\
12 & $(1,3,0)$ & 1.33 & 0 & 0 & 0 & 0.036 & 0.013 & -0.037 \\
13 & $(0,2,1)$ & 0.09 & 0 & 0.005 & -0.049 & 0 & -0.009 & 0.011 \\
14 & $(1,2,1)$ & 0.09 & 0 & -0.005 & -0.007 & 0 & -0.023 & 0.019 \\
15 & $(0,3,1)$ & 1.24 & 0 & 0 & -0.013 & 0.043 & 0.009 & -0.047 \\
16 & $(1,3,1)$ & 2.74 & 0 & 0 & -0.002 & -0.043 & 0.023 & 0.030 \\
\hline Uniform strain : & \multicolumn{7}{c}{$e^{x x}=-0.014, e^{y y}=-0.019, e^{z z}=-0.009$} & \\
\hline \hline
\end{tabular}




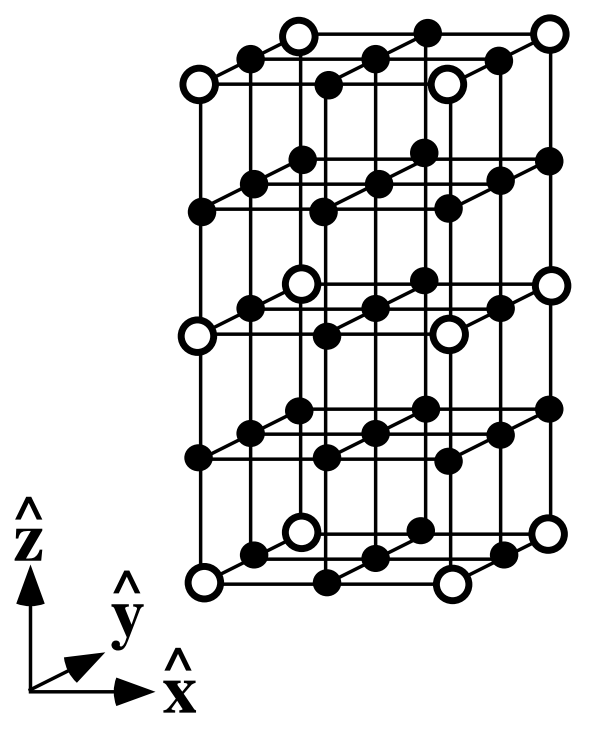

(a)

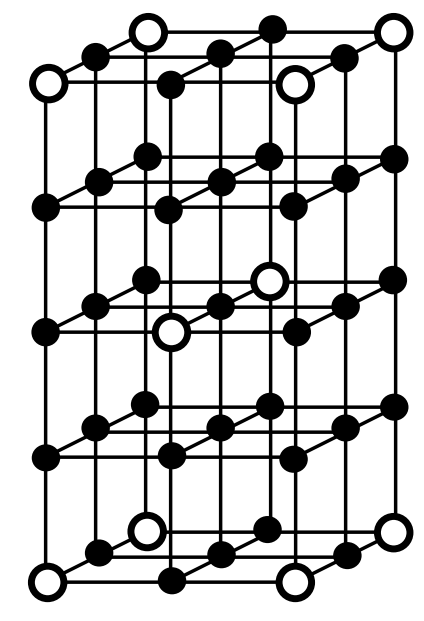

(b)

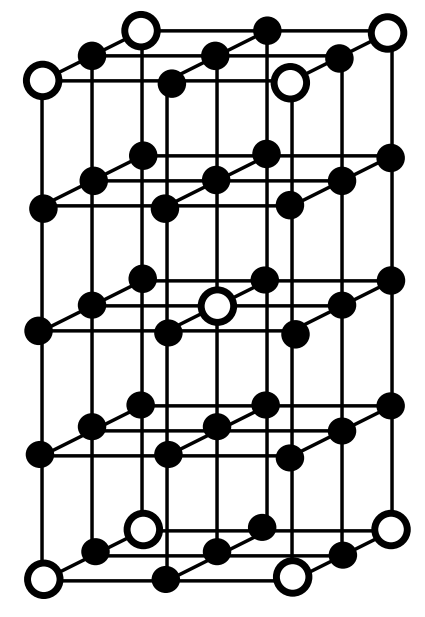

(c)

Figure 1, K. H. Ahn et al. 


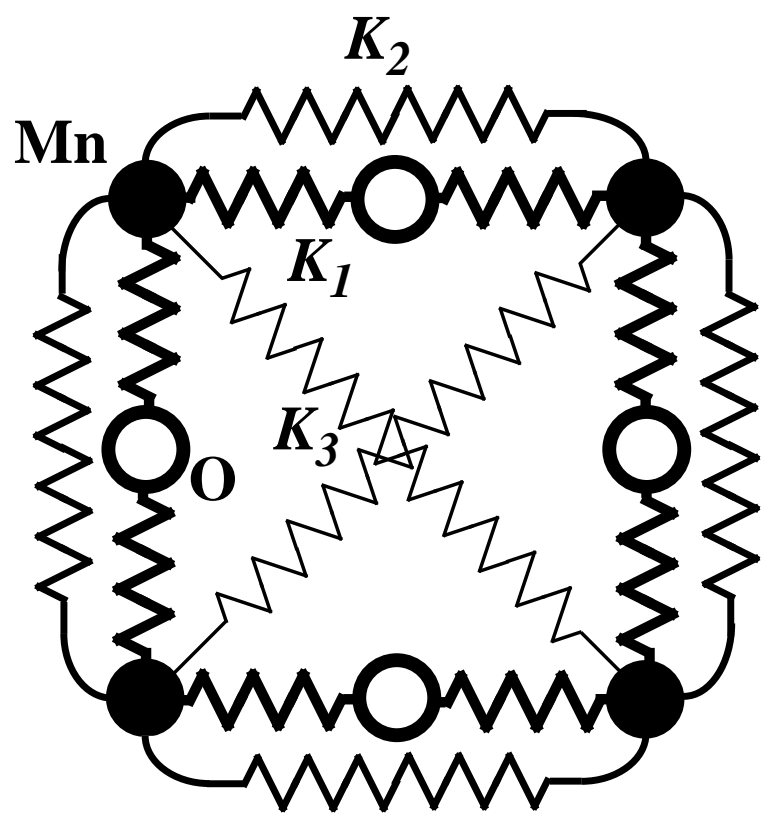

Figure 2, K. H. Ahn et al. 


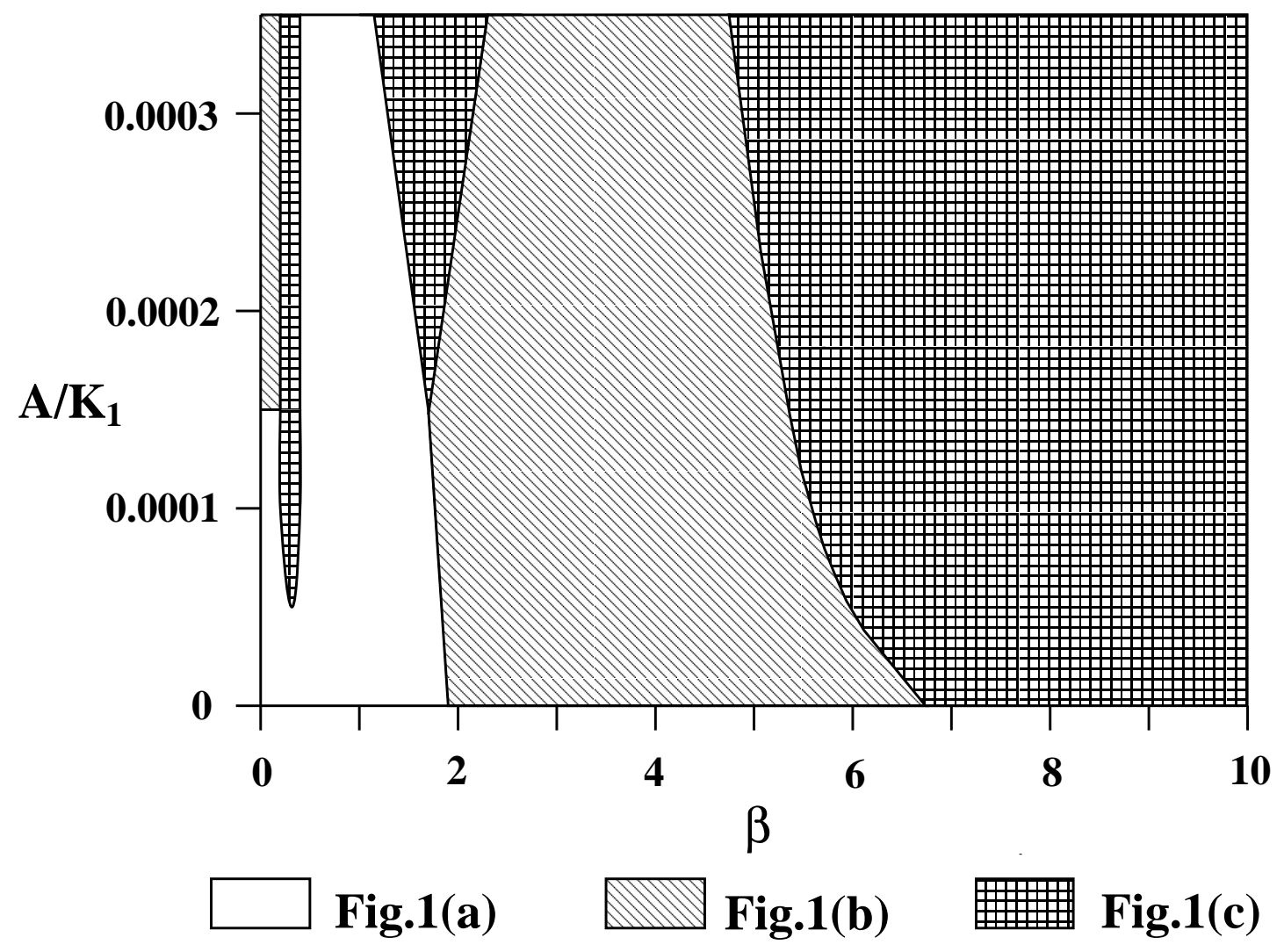

Figure 3, K. H. Ahn et al. 


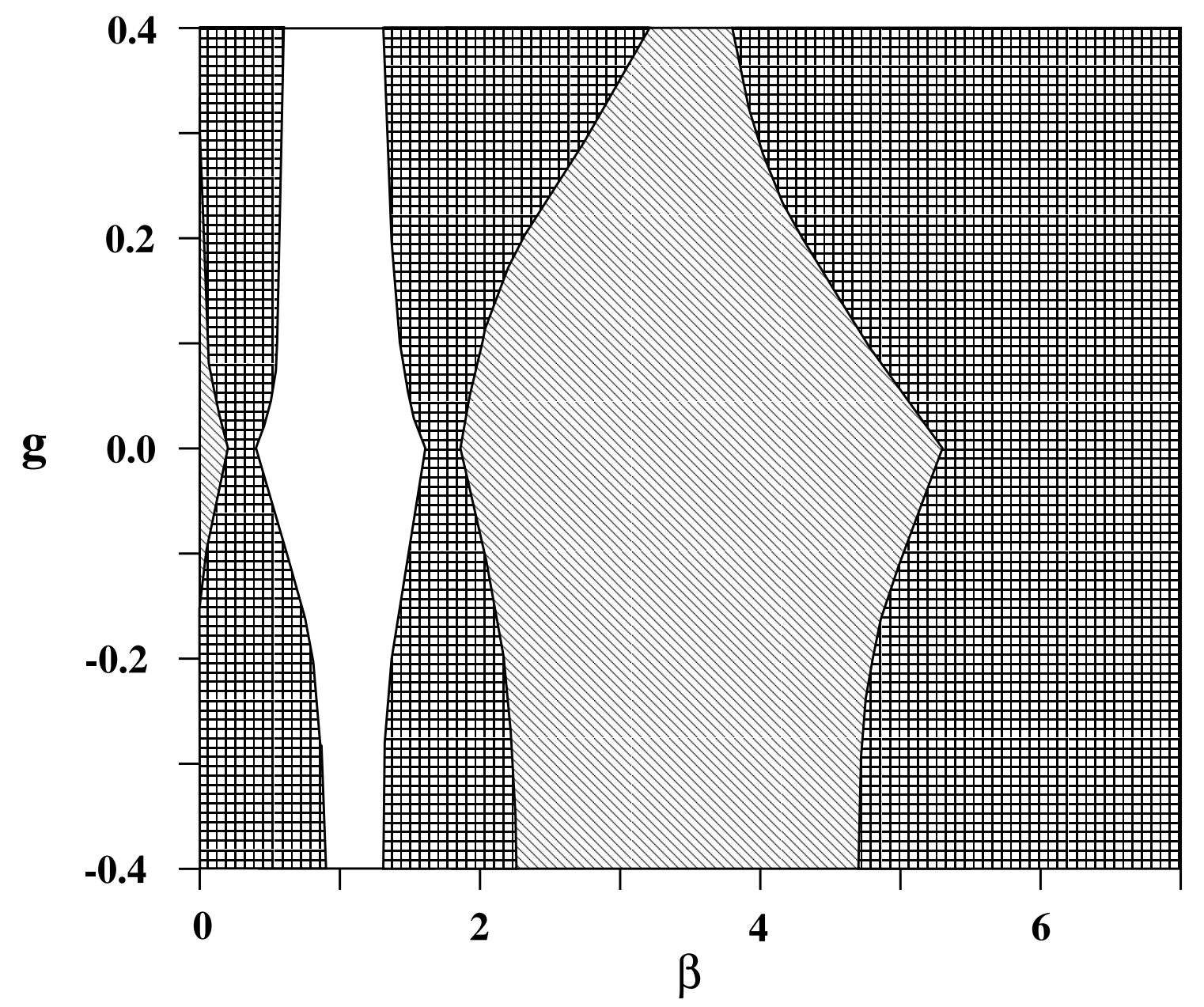

$\square$ Fig.1(a) $\quad$ Fig.1(b) 睓 Fig.1(c)

Figure 4, K. H. Ahn et al. 


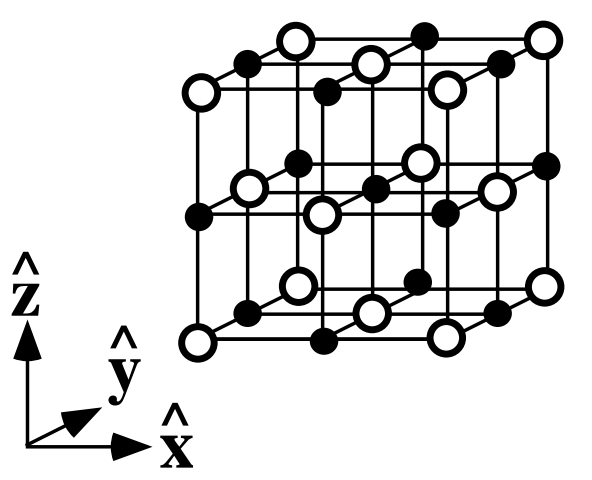

(a)

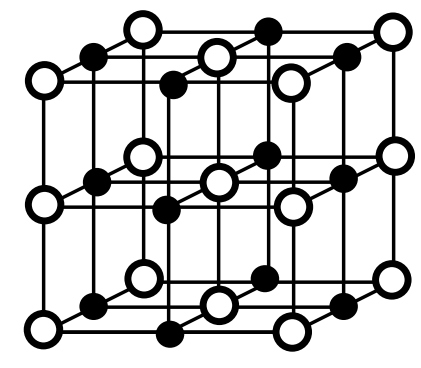

(b)

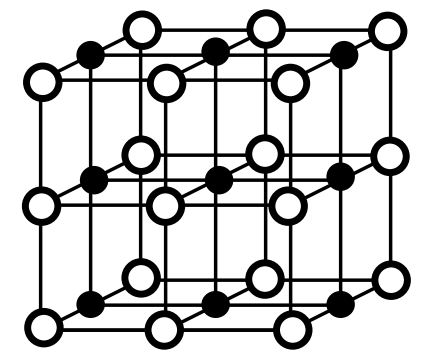

(c)

Figure 5, K. H. Ahn et al. 